\title{
The Feature of Weak Shock Wave Propagated in a Overlong Tunnel
}

\author{
Xiao Hu, Toshiyuki Aoki, Naoya Tokura \\ Graduate School of Engineering Science, Kyushu University, Fukuoka, Japan \\ Email: aoki.toshiyuki.418@m.kyushu-u.ac.jp
}

Received September 19, 2012; revised November 1, 2012; accepted November 10, 2012

\begin{abstract}
Many experiment researches have been developed before. But most of them were carried out with the condition that the tunnel's ratio of length and diameter (x/D) is under 1000. Recently, the process that compression wave convents into shock wave in the overlong tunnel has also been paid attention. In this paper, features of shock wave as it propagates through a overlong tunnel is investigated, rupturing thin films at the entrance to obtain three kinds of shock wave with different intensities. Then study their features respectively during they propagates through a overlong tunnel with $\mathrm{x} / \mathrm{D}$ over 6000 at most. Comprehend shock wave more deeply by comparing the results of experiments.
\end{abstract}

Keywords: Shock Wave; Compression Wave; Micro-Pressure Wave; Overlong Tunnel; Pipes

\section{Introduction}

When compressible fluid in the pipeline is compressed, it will become compression wave and propagates in the pipeline [1]. During the propagation, many noisy problems that affect our health in daily life would appear. For instance, as the engine of the automobile with high output power is turning round in high speed, the airflow from the gas vent's exit will shake substances around such as plate metals. It will occur extremely piercing noise.

Another example is the process that a high-speed train passes through the tunnel [2]. As it is shown in Figure 1, the train like a plunger compresses the air in the tunnel before the it entrances, then an acoustic wave called compression wave is produced and propagates in the tunnel. As it arriving the exit, a part of the flow reverberates back to the tunnel and the rest of it is emitted to the diffusion space which named micro-pressure wave phenomenon [3]. The wave's frequence is lower than $20 \mathrm{~Hz}$ so that it couldn't be heart by people. In many tunnels, micro-pressure wave is just noise of small amplitude that doesn't exert any influence. But in the case of the train whose speed crossing $200 \mathrm{~km} / \mathrm{h}$, the sound pressure level would reach 140 - $150 \mathrm{~dB}$. The disturbance, which can shake doors and windows of nearby buildings, has taken many problems to the daily life of the people there.

Especially in 2027, the bullet train of Japan will exceed a speed of $580 \mathrm{~km} / \mathrm{h}$. shock wave will occur as the train entrances the deep underground tunnel in Tokyo and will propagates through the long tunnel. It became more urgent for researchers to resolve this problem. To cripple the influence from micro-pressure wave, it is necessary to study characteristics of compression wave and shock wave during they propagates in the tunnel.

\section{Experiment}

\subsection{Experiment System}

The experiment system is shown in Figure 2. It is constituted by 4 parts: drive system (hyperbaric chamber), film rupture system, propagates system (low pressure chamber) and measure system. Both of the drive system and the propagates system consist of smooth copper pipes with the external diameter of $20 \mathrm{~mm}$ and internal diameter of $16 \mathrm{~mm}$. The length of the drive system is about $50 \mathrm{~m}$, and the propagates system is over $95 \mathrm{~m}$, to weaken the influe of reflection. Rings connect those systems and thin film composed by ethyl acetate, vinyl acetate, ethanol, is placed between them.

The measure system gets experiment data from pressure sensors (XCS-190). The pressure sensor will induce the change of pressure and transform it into voltage data in order to analyze the result easily. Regard the place $0.75 \mathrm{~m}$ away from thin film as original point $(\mathrm{x} / \mathrm{D}=0)$, and place five pressure sensors named S1 - S5 behind it.

\subsection{Experiment Method}

First, place thin film between the hyperbaric chamber the 
low pressure chamber. A couple floors of film could be piled up if we need stronger shock wave. Inject air into the hyperbaric chamber till the film break up and shock wave begins to propagate during the pipe. As a result of the change of pressure, $\mathrm{S} 1$ - S5 sensors start to get data and send to memory recorder.

\section{Results and Discussion}

\subsection{The Attenuation of Shock Wave from the Experimental Result Compare to the Calculation}

Define $\Delta P_{s}$ as the pressure strength of each testing point and $\Delta P_{s, i}$ as original point's $(\mathrm{x} / \mathrm{D}=0)$. Mirels [4] has achieved the calculation equation about $\Delta P_{s}$ in both laminar and turbulent flow condition, supposing the Much number is smaller than 1. Using the equation, the attenuation of shock wave $\Delta P / \Delta P_{s, i}$ could be indicated as:

Laminar flow condition

$$
\frac{\Delta P_{s}}{\Delta P_{s, i}}=1-\frac{\frac{8}{\sqrt{\pi\left(\gamma_{1}+1\right)}}\left(1+\frac{\gamma_{1}-1}{\sqrt{\sigma_{1}}}\right)}{\sqrt{\frac{U_{s}}{u_{2}} \frac{a_{2} D}{v_{1}}}} \sqrt{\frac{x}{D}}
$$

Turbulent flow condition

$$
\frac{\Delta P_{s}}{\Delta P_{s, i}}=1-\frac{\frac{0.115}{2\left(\gamma_{1}+1\right)^{0.2}}}{\left(\frac{U_{s}}{u_{2}}\right)^{1.4}\left(\frac{D a_{2}}{v_{2}}\right)^{0.2}}\left(\frac{x}{D}\right)^{0.8}
$$

where $\gamma_{1}$ is the specific heat ratio, $\sigma_{1}$ is the Prandtl number, $v_{2}$ is the dynamic viscosity, $u_{2}$ is velocity of flow behind shock wave, $U_{s}$ is the propagation velocity of shock wave and $a_{2}$ is the acoustic velocity behind shock wave.

Using these equations, the attenuation of shock wave from the experimental result compare to the calculation could be showed as Figure 3.

\subsection{The Attenuation of Shock Wave as It Propagated in Pipes}

Make three species of shock wave different in strength of pressure at the original point, named condition $\mathrm{A}, \mathrm{B}$ and $\mathrm{C}$ and the initial values of them increase progressively. The attenuation of these three kinds of shock wave is showed in Figure 4.

Assuming the attenuation of shock wave $\Delta P_{s} / \Delta P_{s, i}$ could be defined by this equation:

$$
\frac{\Delta p_{s}}{\Delta p_{s, i}}=\exp \left(-k \frac{x}{D}\right)
$$

where $k$ is the attenuation coefficient. The bigger it is, the faster shock or compression wave decrease in the pipes. By least square method to get the attenuation coefficient and show them in Figure 5.

It could be observed in Figure 5, when the original shock wave becomes stronger, the attenuation coefficient will also increase.

Moreover, while shock wave regresses to compression wave, the maximum gradient of pressure will change as it is showed in Figure 6. Define non-dimensional maximum gradient as:

$$
\left(\frac{\mathrm{d}(\Delta P)^{\prime}}{\mathrm{d} t^{\prime}}\right)_{\max }=\frac{\sqrt{\kappa} D}{a P_{0}}\left(\frac{\mathrm{d}(\Delta P)^{\prime}}{\mathrm{d} t^{\prime}}\right)_{\text {max }}\left(\mathrm{d}(\Delta P)^{\prime} / \mathrm{d} t^{\prime}\right)_{\text {max }}
$$

where $\kappa$ is the specific heat ratio of flow, $P_{0}$ is the strength of barometric pressure, $a$ is the acoustic velocity.

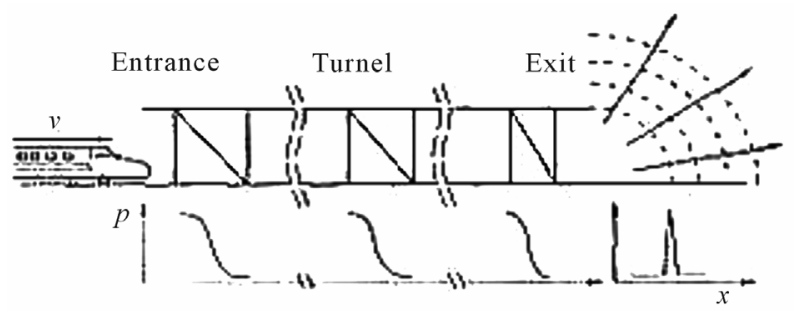

Figure 1. Developmental mechanism of micro-pressure wave.

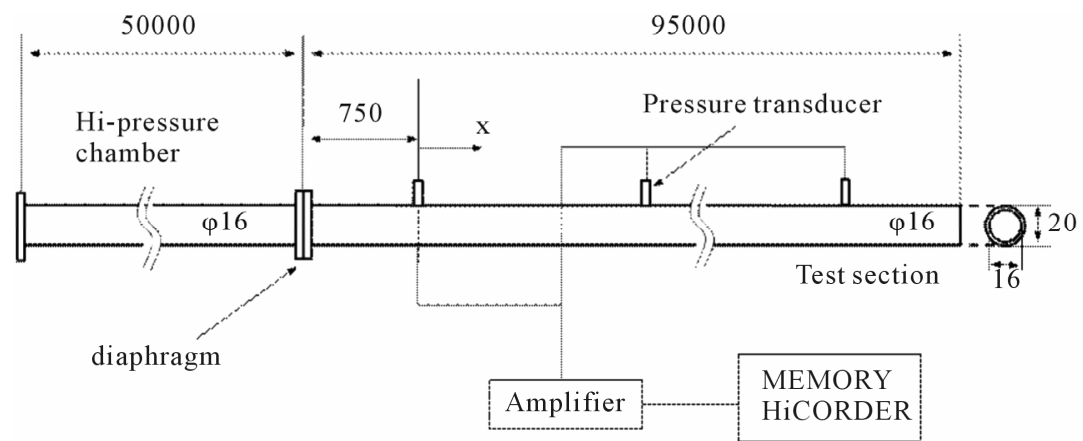

Figure 2. The experiment system. 


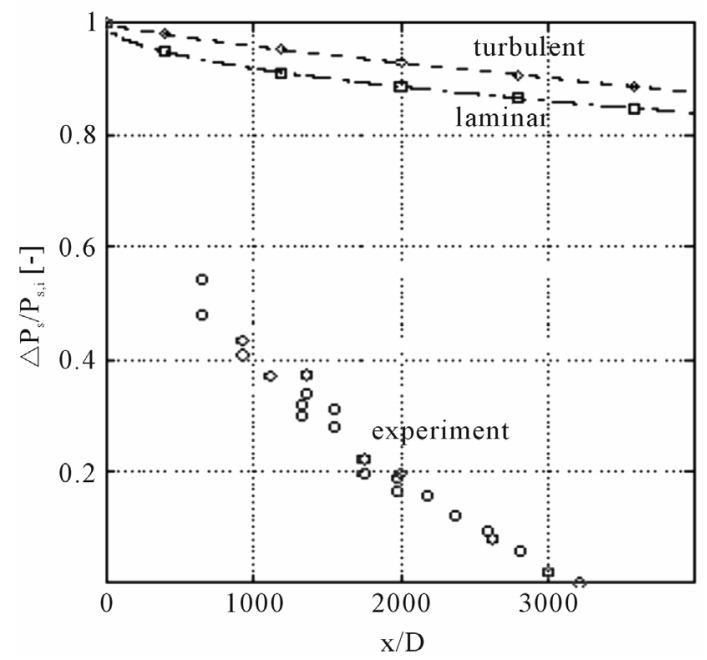

Figure 3. The attenuation of shock wave from the experimental result compare to the calculation.

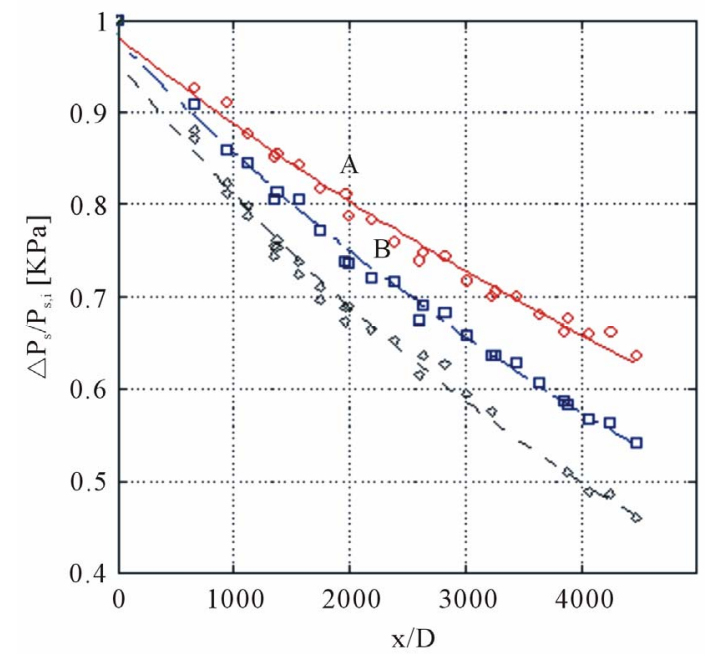

Figure 4. The attenuation of shock wave.

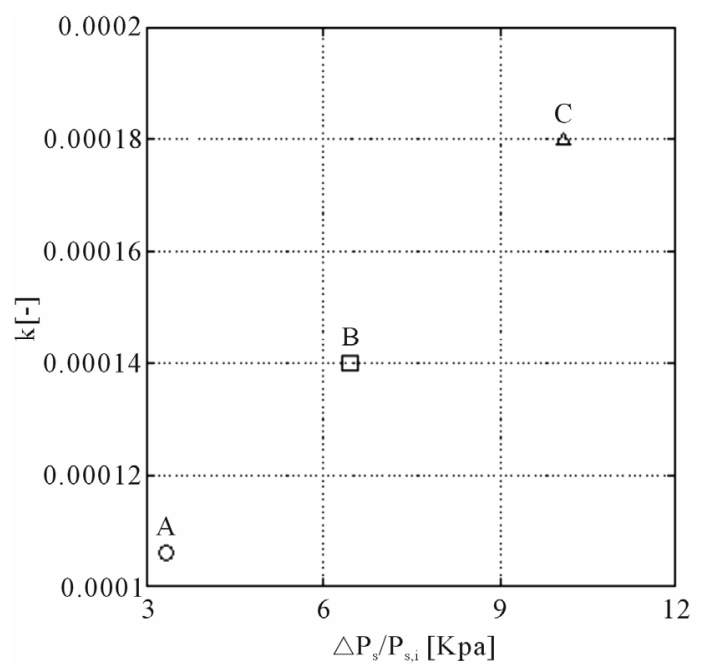

Figure 5. The average attenuation coefficient.

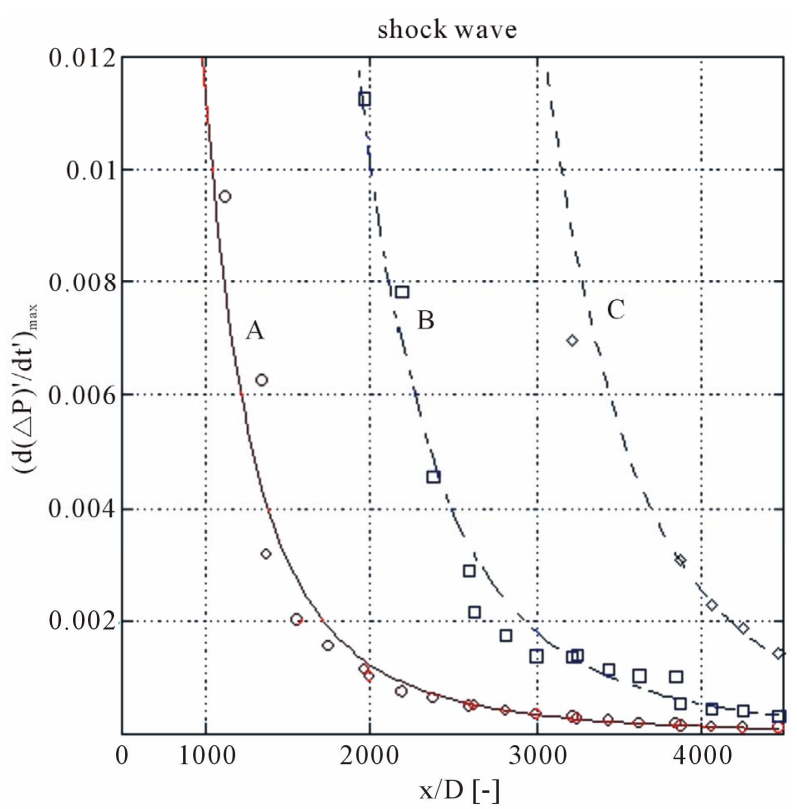

Figure 6. The maximum gradient of pressure.

From Figure 6, it could be observed that results of conditions $\mathrm{A}$ and $\mathrm{B}$ are close to zero finally. It also could be inferred that the result of condition $\mathrm{C}$ will approach zero too. The reason is, as shock wave is propagates in the overlong pipes, viscosity of flow will dominate the attenuation gradually.

\subsection{The Deformation of Wavefront as Shock Wave Propagated in the Overlong Tunnel}

Brown [5] has analyzed the wavefront under the condition of laminar flow Brown:

$$
\begin{aligned}
& \frac{\Delta P_{s}}{\Delta P_{s, i}}=\exp \left(-B T_{0}\right) \operatorname{erfc}\left(\frac{A T_{0}}{2 \sqrt{T-T_{0}}}\right) \\
& A=1+\frac{\gamma_{1}-1}{\sqrt{\sigma_{1}}}, B=1+\frac{\gamma_{1}-1}{\sqrt{\sigma_{1}}}\left(1-\frac{\gamma_{1}}{2 \sqrt{\sigma_{1}}}\right), \\
& T=\frac{4 v_{1}}{D^{2}} t, T_{0}=\frac{4 v_{1}}{D^{2}} \frac{x}{a_{1}}
\end{aligned}
$$

where the implication of these symbols is the same with Equations (1) and (2). Supposing $\mathrm{x} / \mathrm{D}=656$, 1750, 3000,4469 to calculate, and compare with the results of experiment. It is showed in Figure 7.

From the contrast (a) of condition A, it could be observed when $\mathrm{x} / \mathrm{D}$ is 656 , the result of experiment is nearly the same as calculation; but as $\mathrm{x} / \mathrm{D}$ is changed to $1750,3000,4469$, the distinction between them becomes bigger and bigger cause of the nonlinear wave.

Amplify the original strength of shock wave to get Figure 7(b) (Condition B) and (c) (Condition C). It could be observed from this figure as the original strength is 

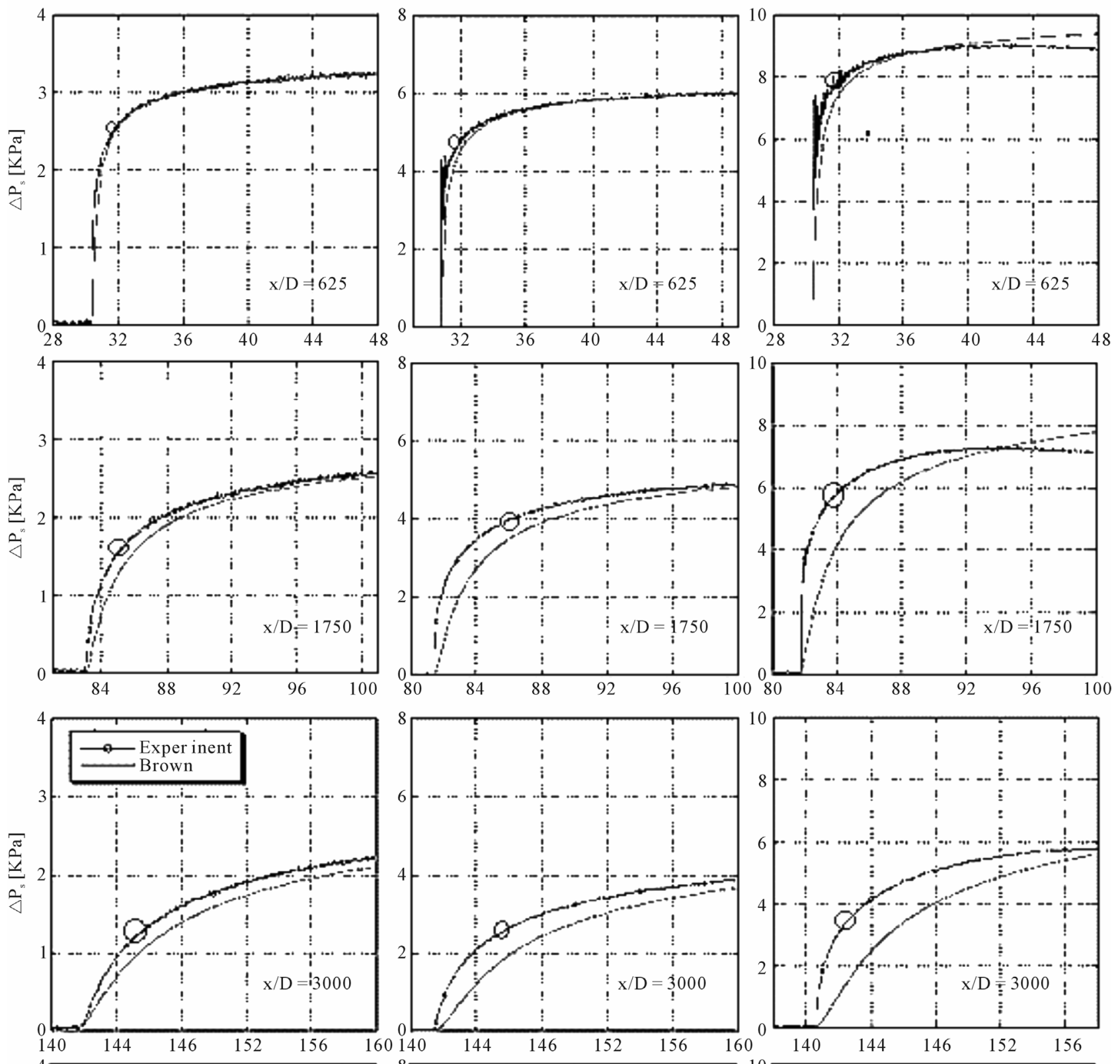

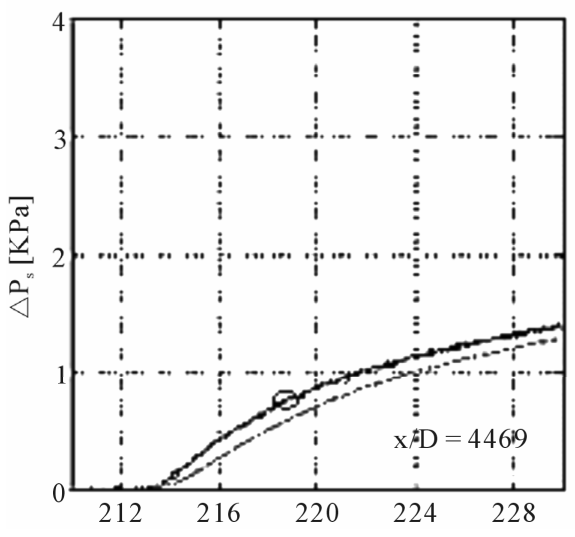

(a)

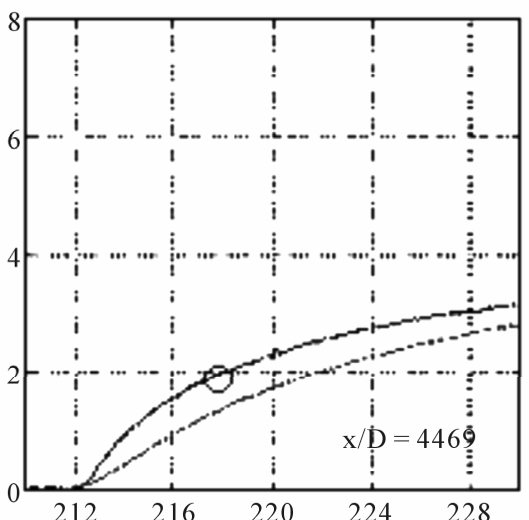

(b)

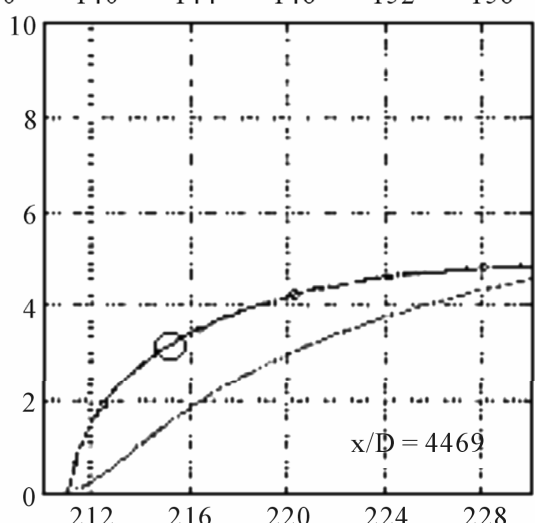

(c)

Figure 7. The deformation of wavefront as shock wave propagated in the overlong tunnel. (a) Condition A; (b) Condition B; (c) Condition C. 
amplified, the distinction between experiment and calculation is more obvious especially (c): while it reaches the maximum value, the strength of wave begins to decrease slowly and in contrast, the calculation increases all through. The reason is as the original strength is amplified, turbulent flow of the non-steady boundary layer behind the wavefront, becomes closer to it and the shear stress makes the pressure loss larger.

\section{Acknowledgements}

- As the original strength of shock wave is amplified, the attenuation becomes larger. It could be observed from the result of experiment, the attenuation's change as distance is proportionality to the original strength.

- After shock wave regressing to compression wave, the maximum pressure gradient abates much more quickly.

- The pressure loss is increasing first. But it reaches the maximum value soon and starts to decrease. It is more obvious as the original shock wave becomes stronger.

\section{REFERENCES}

[1] S. Matsumura, "Shock Wave and Exhaust Noise in the Escape-Pipe of Automobile," Tohoku University Fluid Proseminar No. 4, 1993, p. 113.

[2] A. Yamamoto, "Pressure Variations, Aerodynamic Drag of Train, and Natural Ventilation in Shinkansen Type Tunnel," Quarterly Report of Railway Technical Research Institute (RTRI), Vol. 15, No. 4, 1974, pp. 207-214.

[3] C.-H. Shin and W.-G. Park, "Numerical Study of Flow Characteristics of the High Speed Train Entering into a Tunnel," Mechanics Research Communications, Vol. 30, No. 4, 2003, pp. 287-296. doi:10.1016/S0093-6413(03)00025-9

[4] H. Mirels, "Attenuation in a Shock Tube Due to UnsteadyBoundary-Layer Action (Report/National Advisory Committee for Aeronautics)," US Government Printing Office, Washington DC, 1957, p. 1229.

[5] F. T. Brown, "The Transient Response of Fluid Lines," Journal of Basic Engineering, Vol. 84, No. 4, 1962, pp. 547-553. 